\title{
An examination of user costs in relation to smokers using a cessation service based in the UK
}

Neil Walker ${ }^{1}$, Yaling Yang ${ }^{2 *}$, Vasiliki Kiparoglou, ${ }^{1,2}$, Subhash Pokhrel ${ }^{3}$, Hayley Robinson ${ }^{4}$ and Hugo van Woerden ${ }^{5,6}$

\begin{abstract}
Background: Smoking cessation services provide support to smokers who desire to quit. Published studies to date have looked at the cost and benefit of service provision but typically focus on clinical trial data. Using routinely collected observational data, this study examined the costs involved in providing a service in terms of average health care expenditure per successful quit attempt in addition to population - level cost-effectiveness measures.

Methods: Data were analysed from Quit-51 smoking cessation service across five English regions between March 2013 and March 2016 ( $n=9116)$. For each user, costs were estimated in relation to: (i) time spent with advisers; (ii) prescription of pharmacotherapy. The total costs compared against self-reported quit at 12 weeks, which represents the time period for which the service is offered. Cost per quit (CPQ), with 95\% confidence interval (Cl), was calculated by relating total expenditure to the number of quitters, firstly for the whole dataset and then by subgroups of key categorical variables, namely; gender, age group, the Fagerstrom test for nicotine dependence (FTND) and Index of Multiple Deprivation (IMD). Confidence intervals (Cls) for the mean estimates were derived using a non-parametric bootstrap procedure. Parameters derived from the calculation in relation to treatment were used to estimate potential long-term population outcomes under a scenario where the Quit 51 prescription was rolled out nationally.
\end{abstract}

Results: The overall mean CPQ for this sample as estimated at 12 weeks was $£ 403.51$ ( $95 \% \mathrm{Cl}=£ 393.36$ to $£ 413.76$ ). The estimated CPQs at this time point were comparable for those aged $12-19$ ( $£ 423.56,95 \% \mathrm{Cl}=£ 369.45$ to $£ 492.60)$ and those aged $20-29(£ 430.76,95 \% \mathrm{Cl}=£ 395.95$ to $£ 470.56)$. Differences were also seen in relation to other subgroups considered. The treatment parameters translated to a projected increase of 1.5 quality-adjusted life years (QALYS) per 1000 smokers in the short-term and 23.4 QALYS per 1000 smokers based on a lifetime horizon.

Conclusions: These figures throw light on service expenditure for each successful quit over the timeframe for which the service is offered in addition to highlighting variability in these costs across different subgroups of the user population.

Keywords: Smoking cessation, Cost per quit, NRT, Varenicline

\footnotetext{
* Correspondence: yaling.yang@phc.ox.ac.uk

${ }^{2}$ Nuffield Department of Primary Care Health Science, University of Oxford,

Radcliffe Primary Care Building, Radcliffe Observatory Quarter, Woodstock

Road, Oxford OX2 6GG, UK

Full list of author information is available at the end of the article
} 


\section{Background}

Smoking is a major cause of preventable death and poor health in countries across the world [1]. In particular, smoking is linked to lung and other cancers [2] as well as heart disease, stroke and a range of pulmonary conditions. Bans on smoking in public places, coupled with increasing awareness of the adverse effects on health have helped to reduce the number of smokers in many countries [3]. As well as being beneficial to public health, reducing the prevalence of smoking relieves pressure on health services as a result of the consequent drop in smoking related admissions and achieving further reduction remains a priority for healthcare providers around the world [4].

In the United Kingdom, support is offered to those wishing to quit through the provision of smoking cessation services which operate according to guidelines laid out by the National Centre for Smoking Cessation Treatment (NCSCT) based on the latest available evidence on effective cessation strategies $[5,6]$. Similar services exist in other countries [7]. Smoking services provide access to advice over a number of sessions with a qualified adviser and tailored pharmacotherapy in the form of Nicotine Replacement Therapy, or NRT [8], which is offered for this purpose. Varenicline (proprietary name in UK - Champix) and bupropion (proprietary name in UK - Zyban) are also available, although the last of these is rarely used in light of concerns over side-effects [9].

Of these three treatments, varenicline consistently demonstrates superiority with respect to quit rate based on results from clinical trials $[10,11]$, although alternative treatments not currently available in the UK, e.g. cytisine, may be more effective still [12]. It is less clear whether bupropion is superior to NRT or vice versa, although it is well established that both perform well in relation to placebo [13]. Whilst these findings appear to hold over a number of studies, the projected quit rates and cost effectiveness have not been fully characterised and the decision to prescribe a particular treatment over alternatives may be based on the preference of clinical staff rather than an analysis of client characteristics.

In a climate where healthcare expenditure is increasing in line with new technologies and greater demand, such decisions should be informed by consideration of costs and benefits [14]. Economic models of these smoking cessation interventions from a number of studies indicate that varenicline is the most cost-effective according to various measures such as Quality Adjusted Life Years (QALYs), cost savings and reduction in incidences of smoking-related mortality [15-17]. Results with respect to pharmacotherapy are highly variable when compared across different studies and countries [18]. The costs and benefits used in models of the cost effectiveness of smoking cessation interventions are typically derived from clinical randomised controlled trials (RCT). It is well recognised that the cost-effectiveness of a given intervention can be different in a real-world as opposed to a research context [19-21].

Our primary aim was to calculate the cost per quit (CPQ) in relation to the above treatments using data collected by a smoking cessation service, which affords an insight into the costs in routine clinical practice. We also carried out CPQ calculations across key subgroups in the population of smoking cessation service users. As a secondary aim, figures arising from these calculations, in relation to the three pharmaceutical treatments, were utilised in order to calculate what the long-term populationlevel benefits may be if the Quit 51 programme were rolled out nationally using a freely available tool designed for the calculation of long-term population health parameters, the EQUIPT calculator [22].

\section{Methods}

\section{Smoking cessation services}

Quit 51 offer a smoking cessation service in accordance with National Institute for Health and Care Excellence (NICE) guidelines [6]. The remit of the service is to provide a maximum of 12 sessions of support with an accredited adviser and provision of tailored pharmacotherapy to smokers attempting to quit. A session is typically of 15 min duration although the introductory session will generally take longer in order to cover triaging and discussions around individual background and requirements. Assuming a patient continues with the service for the full duration, they should receive a minimum of 90 min' contact time with an adviser covering a period up to 12 weeks after quitting.

\section{Data}

Data were provided by Quit 51 on patients across six Clinical Commissioning Groups (CCGs) in England for whom the requisite information on treatment and contact time was available. The period covered varied between the different CCGs, but all records fell within the dates 15 March 2013 to 16 March 2016 inclusive (using quit date as the criterion). Best practice was observed in terms of data handling and the project conformed with the principles laid out in the World Medical Association Declaration of Helsinki [23].

At the outset, there were 15,682 records, but a number of restrictions were applied before calculations were made. Observations were removed where: (i) quit date was before subsequent to 9 December 2015, $N=1950$ (ii) smoker recorded as pregnant, $N=802$ (iii) either no or multiple pharmacotherapy was recorded, $N=4254$. The first of steps was taken to ensure as far as possible that all records were complete at the date of data compilation (March 13 2016). Pregnant users of Quit 51 
were not considered here as this subgroup is offered a specialist service which differs in key respects from the standard service. Instances where no or multiple treatment were omitted due to a possibility of misrecording. The above restrictions gave rise to a dataset of 9116 observations derived from 7783 individuals. In order to gain a global perspective on CPQ over this period, repeat observations on individual clients were retained in the calculation. Of the original six clinical regions considered (East Sussex, Sandwell, Surrey, Telford and Wrekin, Walsall, Worcester) only five were used in this calculation as all the Surrey records fell after the exclusion date of 9 December 2015.

\section{Individual costs}

A cost for each individual using the service was estimated based on: (i) pharmacotherapy prescribed; (ii) time spent with an adviser.

With respect to pharmacotherapy, the costs, including prescription and value added tax (VAT) for each treatment were as follows: NRT (combination) - £19.95 per week; Varenicline - $£ 76.80$ per month/ $£ 38.40$ per 2 weeks; and Bupropion - $£ 69.45$ per month/£34.73 per 2 weeks. For clients using NRT, treatment was made available for use to coincide with the agreed quit date. Varenicline, on the other hand, was prescribed between 8 and 14 days prior to the quit date in order to allow for the medical properties of this treatment to take effect. Sometimes an anomaly was observed whereby prescription of these treatments occurred after the 12-week treatment period. Since our inference was based on cessation at 12 weeks, prescription costs were only considered within this timeframe. Table 1 describes how doses were prescribed over time according to treatment pathway. The derivation of medication costs was assessed according to the number of doses prescribed and the individual dose quantities (costs for dose quantities provided above). If, as in some instances, the number of doses indicated in the dataset was above the maximum specified in Table 1 , for the

Table 1 Chronological pattern of medication dose (in weeks) for users of Quit 51 smoking cessation service according to pharmacotherapy prescribed and the prescription route (through pharmacy or other)

\begin{tabular}{lll}
\hline $\begin{array}{l}\text { Treatment } \\
\text { (prescription pathway) }\end{array}$ & $\begin{array}{l}\text { Chronological pattern of dosing } \\
\text { covering 12 weeks of service } \\
\text { use (dose quantity in weeks) }\end{array}$ & $\begin{array}{l}\text { Max. no. } \\
\text { of doses }\end{array}$ \\
\hline NRT (all) & $\begin{array}{l}1+1+1+1+1+1+ \\
1+1+1+1+1+1\end{array}$ & 12 \\
$\begin{array}{l}\text { Varenicline (pharmacy) } \\
\text { Varenicline (any route } \\
\text { other than pharmacy) }\end{array}$ & $2+2+2+2+2+2$ & 6 \\
Bupropion (all) & $2+2+4$ & 4 \\
\hline
\end{tabular}

purposes of the calculation this was fixed at this maximum, so as not to include costs beyond the standard treatment timeframe.

The time spent with an adviser was recorded for each session attended. The figures around adviser time for each session were based on average values, although it is conceivable that a degree of variability exists on a caseby-case basis, for example, in relation to distance travelled by an adviser. The total time spent with an adviser was taken from a variable called "Total Contact Time" in the master database, covering the full 12 weeks of potential engagement with the service. The estimated time was multiplied by an hourly charge of $£ 26.32$, based on the following breakdown of costs: (i) Adviser - $£ 23.65$ per hr.; (ii) Room - $£ 0.20$ per hr.; (iii) Equipment - $£ 0.49$ per hr.; (iv) Travel - $£ 0.74$ per hr.; and (v) Advertising $£ 1.24$ per hr. This itemisation of costs in relation to both pharmacotherapy and counselling was given by the service provider, based on the financial year 2015-2016. Central overhead costs for the service were not included.

\section{Cost-per-quit calculation and confidence intervals}

Using the above method, we derived individual costs up to the full potential 12 weeks' of service use. As an outcome we used 12-week self-reported quit. This was recorded as a binary response ( 0 or 1$)$ where 0 indicated 'failed quit attempt' and 1 indicated 'successful quit attempt'. The average CPQ for this dataset was calculated at this time point as follows:

$$
C P Q=\frac{T}{n_{\text {quit }}}
$$

where $C P Q=$ the average $C P Q$ in the sample, $T=$ the total costs summed across all patients and $n_{\text {quit }}=$ the total number of successful quits (i.e. where binary outcome of quit success $=1$ ). The average $C P Q$ was then also calculated for all categories of the following factors: (i) Age, (ii) Gender, (iii) Treatment, (iv) Fagerstrom test for nicotine dependence (FTND) index [24] and the Index of Multiple Deprivation (IMD) [25]. Age was split into arbitrary categories $(<19,20-29,30-49,50-69$ and $70+$ years) for this purpose.

The FTND index is a score (1-10) reflecting an individual's degree of dependence on cigarettes, based on a number of questions. The lower the score, the lower the dependence inferred. To simplify presentation, this was re-categorised into four groups $(0-3,4-5,6-7$ and $8-10)$ prior to analysis such that each category consisted of a suitable number of records for statistical purposes. The IMD is an area deprivation score mapped to postcode and scored on a scale of 1-10 inclusive, with lower scores indicating greater deprivation. In order to 
facilitate interpretation when referring to a deprivation effect, these scores were inverted so that $1=$ least deprived/most affluent area with increasing score indicating a greater degree of deprivation (maximum $=10$ ). As with FTND, this measure was grouped into suitably large categories prior to the calculation (deprivation $=1-$ $3,4-6,7-8$ and $9-10$ respectively).

Derivation of confidence intervals was complicated by the fact that the distribution of costs was non-standard, in addition to the fact that there were two populations to take into account - successful quits and failed quits. Thus there were different sources of uncertainty to contend with: (i) the costs within both the failed and successful quit subgroups; and (ii) the proportion of successful/failed quits.

To avoid assumptions about the underlying distributions a bootstrap procedure was used to simultaneously deal with both sources of uncertainty. For a given simulation, the original population was sampled with replacement $\mathrm{N}$ times where $\mathrm{N}=$ the number of observations in the population. If a given record was sampled, both the cost and quit status were retained. From this bootstrap sample, a CPQ was calculated as above (dividing the total cost by the number of successful quits). This procedure was repeated 1000 times giving a bootstrap sample from which a $95 \%$ confidence interval was derived with reference to the percentiles at $2.5 \%$ and $97.5 \%$. This procedure was carried out within each subgroup with the value $\mathrm{N}$ being determined by the relevant subgroup sample size.

All the CPQ calculations were carried out in GenStat [26] 18th edition.

In order to extrapolate the short-, medium- and longterm healthcare costs and outcomes (QALYs) of Quit 51, a recently developed return on investment (ROI) model, known as EQUIPTMOD [22], was applied using the treatment parameters used here. The EQUIPTMOD is a European initiative to quantify the utility on investment in protection from tobacco [27] and allows users to simulate the costs and QALYs of smoking cessation interventions under various user-defined scenarios. Details regarding the EQUIPT model and underlying assumptions are described elsewhere [22] and the software is available at the following website (http://equipt.eu).

Extrapolating from the five regions represented in our calculation, a scenario was assumed in which Quit 51 was rolled out to the whole of England. This was then compared against a baseline scenario in which no smoking cessation support was available. The baseline thus represented theoretical gross cost of tobacco smoking in the absence of interventions. The difference in costs (both treatment costs due to smoking attributable diseases and costs of implementing Quit 51 at the national level) and QALYs could then be regarded as the benefit derived from the Quit 51 service. In order to achieve this, the EQUIPTMOD was first run according to a "baseline" setting of no treatment. Then, we added parameters based on the 'Quit 51 approach' to the model via three specific interventions that characterise the Quit 51 service (and Stop Smoking Services more generally), i.e. NRT + support; varenicline + support; and bupropion + support, using the 'customise interventions' functionality of EQUIPTMOD. Three parameters were entered for each treatment arm to model the Quit 51 approach in EQUIPTMOD, namely the uptake rate amongst quitters, the cost per user of the service (regardless of whether the individual has quit or not) and projected relative rate of abstinence (relative to no treatment baseline of $4 \%$ ) at 12 months. The values entered for uptake and cost per head reflected those in our sample, whereas the relapse rates were based on 12-month estimates as used in EQUIPTMOD for users of NRT, varenicline and bupropion (albeit without behavioural support as is provided in the Quit 51 prescription).

\section{Results}

Summary statistics in Table 2 highlight trends in quit rates between different subgroups before we consider results from statistical analysis. Frequency of prescriptions shows that NRT was prescribed for more than $80 \%$ of the clients in this sample and also highlights the rarity with which bupropion was prescribed ( $0.4 \%$ of clients).

The overall average CPQ was $£ 403.59$ (95\% CI = $£ 393.36$ to $£ 413.76$ ) at 12 weeks (Table 3). Differences were observed between age groups such that CPQ tended to decrease with increasing age, although for the two youngest age groups (12-19 and 20-29 years) the average costs were effectively indistinguishable when the CIs are taken into account (mean CPQs; $£ 423.56$ and $£ 430.76$ respectively) as was the case also for the two oldest age groups (mean CPQ $=£ 393.22$ and $£ 390.88$ for the 50-69 and 70+ year age groups respectively). Regarding the pharmacotherapy treatment groups, varenicline use was associated with a slightly higher CPQ than NRT use (mean CPQ $=£ 401.60$ and $£ 413.06$ for varenicline and NRT respectively), but overlapping confidence intervals for these estimates implied insufficient evidence to conclude that this represented a significant difference. The cost per quitter for smokers prescribed bupropion was markedly lower than for the other two treatments (mean CPQ and 95\% CI; £226.56, $£ 177.21$ to $£ 314.21$ ).

Our calculations showed an elevated average cost for females relative to males (mean CPQ $=£ 412.67$ and $£ 393.97$ respectively) with the confidence intervals indicating that this difference was statistically significant.

The costs steadily increased in line with increased tobacco dependence (mean CPQ $=£ 378.87, £ 411.78$, 
Table 2 Summary statistics for key variables in the sample based on quit rate at 12 weeks $(N=9116)$

\begin{tabular}{|c|c|c|c|}
\hline & & $N(\%)$ & $\mathrm{N}$ quit at 12 weeks (\%) \\
\hline \multirow[t]{5}{*}{ Age $($ mean $=44.8 \mathrm{yrs})$} & $12-19$ yrs & $509(5.6 \%)$ & $116(22.8 \%)$ \\
\hline & $20-29$ yrs & $1189(13.1 \%)$ & 296 (24.9\%) \\
\hline & $30-49$ yrs & $3911(43.0 \%)$ & $1262(32.3 \%)$ \\
\hline & $50-69$ yrs & $2955(32.5 \%)$ & $1068(36.1 \%)$ \\
\hline & $70+y r s$ & $538(5.9 \%)$ & 192 (35.7\%) \\
\hline \multirow[t]{2}{*}{ Gender } & Male & $4249(46.6 \%)$ & $1425(33.5 \%)$ \\
\hline & Female & 4867 (53.4\%) & $1510(31.0 \%)$ \\
\hline \multirow[t]{3}{*}{ Treatment } & NRT & $7373(80.1 \%)$ & $2117(28.7 \%)$ \\
\hline & varenicline & $1708(18.7 \%)$ & 799 (46.8\%) \\
\hline & bupropion & $35(0.4 \%)$ & $19(54.3 \%)$ \\
\hline \multirow[t]{4}{*}{ FTND $($ Mean $=4.89)$} & $0-3$ & $1534(26.2 \%)$ & $622(40.5 \%)$ \\
\hline & $4-5$ & $1884(32.2 \%)$ & 727 (38.6\%) \\
\hline & $6-7$ & $1676(28.6 \%)$ & 641 (38.2\%) \\
\hline & $8-10$ & $766(13.1 \%)$ & $236(30.8 \%)$ \\
\hline \multirow[t]{4}{*}{ Deprivation $($ Mean $=7.22$ ) } & $1-3$ & $886(10.8 \%)$ & 319 (36.0\%) \\
\hline & $4-6$ & $1838(22.4 \%)$ & $635(34.5 \%)$ \\
\hline & $7-8$ & $2157(26.3 \%)$ & $698(32.4 \%)$ \\
\hline & $9-10$ & $3321(40.5 \%)$ & $1180(35.5 \%)$ \\
\hline
\end{tabular}

Table 3 Average cost per quit (with approximate 95\% Cl) calculated at the 12 week time point, with supporting information

\begin{tabular}{|c|c|c|c|c|c|c|c|}
\hline \multirow[t]{2}{*}{ Variable } & \multirow[t]{2}{*}{ Levels } & \multicolumn{6}{|c|}{12 weeks } \\
\hline & & $\mathrm{N}$ & Total cost $(£)$ & Cost per head $(£)$ & $\mathrm{N}$ quits & Quit rate $(\%)$ & Mean CPQ $(95 \% \mathrm{Cl})(£)$ \\
\hline \multirow[t]{5}{*}{ Age } & $12-19$ & 509 & 49,133 & 96.53 & 116 & 22.79 & $423.56(369.45,492.60)$ \\
\hline & $20-29$ & 1189 & 127,504 & 107.24 & 296 & 24.89 & $430.76(395.95,470.56)$ \\
\hline & $30-49$ & 3911 & 512,284 & 130.99 & 1262 & 32.27 & $405.93(391.53,423.23)$ \\
\hline & $50-69$ & 2955 & 419,963 & 142.12 & 1068 & 36.14 & $393.22(378.45,409.60)$ \\
\hline & $70+$ & 538 & 75,050 & 139.50 & 192 & 35.69 & $390.88(357.16,427.00)$ \\
\hline \multirow[t]{2}{*}{ Gender } & Male & 4249 & 561,404 & 132.13 & 1425 & 33.54 & $393.97(380.36,407.97)$ \\
\hline & Female & 4867 & 623,127 & 128.03 & 1510 & 31.03 & $412.67(398.41,427.01)$ \\
\hline \multirow[t]{3}{*}{ Treatment } & NRT & 7373 & 850,191 & 115.31 & 2117 & 28.71 & $401.60(389.91,414.63)$ \\
\hline & varenicline & 1708 & 330,035 & 193.23 & 799 & 46.78 & $413.06(395.16,433.55)$ \\
\hline & bupropion & 35 & 4305 & 122.99 & 19 & 54.29 & $226.56(177.12,314.21)$ \\
\hline \multirow[t]{4}{*}{ FTND } & $0-3$ & 1534 & 236,567 & 153.62 & 622 & 40.55 & $378.87(359.35,399.99)$ \\
\hline & $4-5$ & 1884 & 299,362 & 158.90 & 727 & 38.59 & $411.78(393.08,433.31)$ \\
\hline & $6-7$ & 1676 & 270,188 & 161.21 & 641 & 38.25 & $421.51(401.06,444.88)$ \\
\hline & $8-10$ & 766 & 120,191 & 156.91 & 236 & 30.81 & $509.28(464.80,562.87)$ \\
\hline \multirow[t]{4}{*}{ Deprivation } & $1-3$ & 886 & 136,039 & 153.54 & 319 & 36.00 & $426.45(396.92,460.57)$ \\
\hline & $4-6$ & 1838 & 266,099 & 144.78 & 635 & 34.55 & $419.05(398.11,443.28)$ \\
\hline & $7-8$ & 2157 & 300,324 & 139.23 & 698 & 32.36 & $430.26(408.13,451.16)$ \\
\hline & $9-10$ & 3321 & 451,353 & 135.91 & 1180 & 35.53 & $382.50(369.26,397.69)$ \\
\hline
\end{tabular}


$£ 421.51$ and $£ 509.88$ for binned categories of FTND; $1-3,4-5,6-7$ and $8-10$ respectively). The trend with respect to IMD scores was not obviously linear. The CPQ for the first 3 groups, in ascending order of deprivation, were broadly similar (£426.45, £419.05 and $£ 509.88$ for deprivation score groups of $1-3,4-6$ and 7-8 respectively). In contrast, the average CPQ for the group of highest deprivation (inverted IMD = 9-10) was $£ 382.50$, significantly lower than all others.

Table 4 reports the results from the analysis based on the EQUIPTMOD. Parameters entered to simulate a Quit 51 prescription were as follows; uptake rate $80.1 \%, 18.7 \%$ and $0.4 \%$ (see Table 2); cost per user $£ 115.45, £ 190.16$ and $£ 122.99$ for NRT (see Table 3); relative success rate (against baseline of $4 \%$ cessation) $2.14,2.24$ and 1.6, for varenicline and bupropion respectively in each case [4]. The figures are presented to cover various time horizons -2 years (short term); 5-10 years (medium term) and lifetime horizon (long term). The Quit 51 approach produced an average of 35.21 quitters per 1000 smokers. This translated to health gains of 1.5 QALYs per 1000 clients in the short term to 23.4 QALYs per 1000 clients using a lifetime horizon. Although the cost per QALY gain in the first 2 years of Quit $51(£ 58,736)$ is well above the NICE threshold of $£ 20,000$ per QALY, this intervention would become cost-effective in the medium term and would be highly cost-effective in the long term, compared with the counterfactual scenario. For every $£ 1$ invested in Quit 51 , over a lifetime horizon one would expect a return of $65 p$ just in healthcare savings (i.e. savings coming from treating fewer cases of smoking attributable diseases) but this would increase to a more substantial $£ 4.22$ if QALY gains are considered from a societal perspective.

\section{Discussion}

In this study we have used observational data recorded by a smoking cessation service to gain an understanding of the typical real world CPQs from a service or NHS perspective. From these data, we estimate the average cost per quitter at $£ 403.59$, with a significant degree of variation seen across certain subgroups of the client population. These calculations were based on applying an average rate with respect to adviser time and a more accurate picture may be obtained where more detailed individual-level information exists. Furthermore, Quit 51 overhead costs were not included in addition to there being no allowance made for costs to the individual of service use (travel and parking costs etc.). Thus the figures presented here should be interpreted strictly in relation to costs associated with provision of medication and behavioural support by stop smoking services.

Age-based results imply greater difficulty amongst the young (teenagers and the 20-29 year old group in this instance) in quitting, as has been reported elsewhere [28]. This age group is an important one to reach and support, given that the long term benefits of cessation are greater at this stage of life than amongst those of an older age [1]. Analysis elsewhere has shown CPQ figures for different types of internet and mobile phone smoking cessation assistance [29] are favourable in relation to those presented here. Given widespread use of internet technology amongst the young in general, this represents an appealing alternative as a strategy for driving down quit rates (and associated costs) with users of the service in this age group.

These data show that average cost per quit amongst males was slightly reduced relative to females, a finding which is consistent with men being more likely to quit in the context of smoking cessation services $[28,30]$, although the magnitude of the difference was not great when considered against the overall average costs observed (difference $=£ 18.70$ at 12 weeks). If the reason behind the different quit rates between men and women was related to anxiety about weight gain due to quitting smoking, the provision of a more comprehensive service combining both smoking cessation and weight loss may be worth considering [31].

Although varenicline users had a higher quit rate than those using NRT, this didn't translate to a lower CPQ,

Table 4 Short-, medium- and long-term costs and outcomes of the Quit 51 approach modelled using the EQUIPTMOD model [22] based on 35.21 quitters per 1000 smokers

\begin{tabular}{|c|c|c|c|c|}
\hline \multirow{2}{*}{ Metrics } & \multicolumn{4}{|c|}{ Time horizon } \\
\hline & 2 years & 5 years & 10 years & Lifetime \\
\hline Average healthcare costs (per smoker) & $£ 862$ & $£ 2091$ & $£ 3944$ & $£ 11,608$ \\
\hline Average total costs (per smoker)(includes cost of intervention) & $£ 957$ & $£ 2186$ & $£ 4039$ & $£ 11,704$ \\
\hline Average QALYs (per smoker) & 1.57 & 4.53 & 6.57 & 14.81 \\
\hline QALY gains (per 1000) ${ }^{\mathrm{a}}$ & 1.5 & 3.7 & 7.5 & 23.4 \\
\hline Cost/QALYa & $£ 58,736$ & $£ 19,756$ & $£ 7582$ & $£ 402$ \\
\hline Benefit-Cost ratio (healthcare savings only) & 0.07 & 0.16 & 0.29 & 0.65 \\
\hline Benefit-Cost ratio (healthcare savings + QALY gains valued at £20,000/QALY) & 0.29 & 0.73 & 1.44 & 4.22 \\
\hline
\end{tabular}

${ }^{\mathrm{a} C o m p a r e d ~ w i t h ~ a ~ b a s e l i n e ~ s c e n a r i o ~ o f ~ n o ~ i n t e r v e n t i o n ~}$ 
which was slightly higher for varenicline than NRT. Models and analysis looking into ICERs and QALYs elsewhere suggest varenicline is more cost-effective than NRT $[15,16,32]$, thus contrasting to a degree with the findings in this analysis. The estimated CPQ for bupropion was perhaps surprisingly low, although it should be remembered that the standard course of treatment for bupropion users only amounts to three prescriptions to cover 8 weeks in contrast to NRT and varenicline, which are prescribed to cover a period of 12 weeks (see Table 1). Even allowing for this, the CPQ for bupropion was strikingly low which contrasts with results in the literature. Nonetheless, the sample for this subgroup was very low and thus associated figures should be considered indicative only.

Greater CPQ with increased nicotine dependence is a logical result of greater difficulty quitting with increased dependence. No obvious trend was evident in relation to the deprivation groups although it was notable that the lowest CPQ was observed in relation to the most deprived group. It is possible to speculate that the higher prevalence of smoking in the most deprived population may mean that there is a higher proportion in this group who have low levels of addiction compared to other social groups, where those who can easily give up have already done so and one is left with a rump of smokers who find quitting very difficult.

In this preliminary work, we have only considered CPQ across subgroups of individual factors without considering how they might covary together (e.g. age and gender). Exploratory work indicated that some kind of interaction may be present between gender and age group, but this was not within the scope of the current preliminary presentation. Nonetheless, this could provide the basis of future work, particularly if more detailed individual costings become available.

In this analysis, the calculation of CPQ was primarily restricted to a health service perspective. However, the true cost of quitting smoking also involves the smoker's time and travel to and from service venues, and other indirect costs such as leave from work or provision of childcare. Therefore, the true cost to individuals and society is likely to be greater than the figures we have presented here.

Our analysis was based on routinely collected service data. It was necessary to exclude a proportion of the data before the calculation was carried out. In the case of exclusion on the basis of (i) date and (ii) quitter recorded as pregnant this was a practical necessity. In addition, some records were omitted as either no or multiple treatment was recorded (4254 of the original 15,682 records fell into this category). This raises the issue of data quality when dealing with data of this nature and potential bias introduced due to excluded and missing records. Furthermore, we have assumed the five CCGs considered are approximately representative of England as a whole. It is difficult to quantify the resulting impact but the large sample size provides some reassurance regarding robustness in these results. Furthermore, the bootstrapped confidence intervals allowed us to capture quantifiable uncertainty with a non-standard distribution.

Costings were based on figures provided by Quit- 51 as of the financial year 2015-2016 and the results should be interpreted on this basis. The impact of inflation over this period could have been taken into account, but should not have greatly altered the findings, particularly in regard to relative differences between the subgroups of interest, as the inflation rate was low over the period of this study.

Our cost per QALY findings are subject to the limitations of EQUIPTMOD which have been described in Coyle et al. [22]. We made use of the available model to predict the value of Quit 51 against a baseline scenario of no intervention to make meaningful predictions - this assumption may however be an important limitation in itself. Information on quit rates at 52 weeks was recorded sparingly in this dataset and therefore we used the quit rates adopted within EQUIPTMOD. This means that we have assumed relapse rates of approximately $70 \%, 81 \%$ and $88 \%$ for the NRT, varenicline and bupropion treatment arms respectively subsequent to the observed 12-week quit rates in Quit 51. Thus these figures are based on a number of assumptions, but nonetheless serve to demonstrate how parameters derived from our calculations can be used to estimate long-term population level cost-benefit outcomes.

\section{Conclusions}

This work has highlighted differences in CPQ between different demographic subgroups, most notably increased expense in relation to teenage and 20-29 year old clients, female users and those reporting greater nicotine dependence at baseline. Such information could be factored into service provision, based on user population characteristics. The higher costs associated with treating teenagers are particularly important from a service planning perspective. The CPQ for varenicline was higher than that for NRT and bupropion, which is in contradiction to some other studies. The data presented in this study may contribute to future meta-analyses to resolve the relative $\mathrm{CPQ}$ in real life situations.

\section{Abbreviations}

CCG: Clinical commissioning group; Cl: Confidence interval; CPQ: Cost per quit; FTND: Fagerstrom test for nicotine dependence; IMD: Index of multiple deprivation; NCSCT: National centre for smoking cessation treatment; NICE: National institute for health care and excellence; NRT: Nicotine replacement therapy; QALY: Quality adjusted life year; RCT: randomised controlled trial; VAT: Value added tax 


\section{Acknowledgements}

The authors are grateful to Quit 51 for providing free access to the data used in this research. The EQUIPTMOD was developed by the EQUIPT Study Group with European Commission's funding (FP7) and the model is available to download freely from http://equipt.eu. This work was supported by the NIHR Oxford Biomedical Research Centre.

\section{Funding}

Dr. Vasiliki Kiparoglou, Dr. Yaling Yang and Dr. Neil Walker were funded by the NIHR Oxford Biomedical Research Centre.

\section{Availability of data and materials}

The data may be obtained from the authors for academic purposes, subject to appropriate Caldicott Guardian and data protection requirements being met.

\section{Authors' contributions}

NW carried out cost-per-quit analyses, wrote the initial draft and carried out revisions in discussion with co-authors. HvW, HR, VK and YY contributed to analytical design and made substantial contributions to the original and revised drafts. HR provided advice on practical aspects of smoking cessation services in North 51. NW ran the original EQUIPT model via its interface with guidance from SP who conducted a secondary analysis and made significant written contributions to parts of Methods and Results relating to the EQUIPT model. All authors read and approved the final manuscript.

\section{Ethics approval and consent to participate}

Users of the Quit-51 service are informed at initial registration that data recorded in relation to their cessation attempt may be used for research purposes. Consent was provided by Quit-51 for this data to be used in analysis.

\section{Consent for publication}

Not applicable.

\section{Competing interests}

$\mathrm{SP}$ is associate editor of BMC Health Service Research. HR works for Quit-51 who provided the data which were analysed in this work. Apart from this, the authors declare no competing interests.

\section{Publisher's Note}

Springer Nature remains neutral with regard to jurisdictional claims in published maps and institutional affiliations.

\section{Author details \\ 'NIHR Oxford Biomedical Research Centre, Churchill Hospital, Old Road, Headington, Oxford OX3 7LE, UK. ${ }^{2}$ Nuffield Department of Primary Care Health Science, University of Oxford, Radcliffe Primary Care Building, Radcliffe Observatory Quarter, Woodstock Road, Oxford OX2 6GG, UK. ${ }^{3}$ Health Economics Research Group, Institute of Environment, Health and Societies, Brunel University, Uxbridge UB8 3PH, UK. ${ }^{4}$ Quit 51 Stop Smoking Service, Burton-on-Trent DE14 2AZ, UK. Institute of Primary Care \& Public Health, Cardiff University, Cardiff, UK. ${ }^{6}$ Centre for Health Science, University of the Highlands and Islands, Inverness IV2 3JH, UK.}

\section{Received: 31 December 2016 Accepted: 7 March 2018} Published online: 15 March 2018

\section{References}

1. Jha P, Peto R. Global effects of smoking, of quitting, and of taxing tobacco. N Engl J Med. 2014;370(1):60-8

2. Pinsky PF, Church TR, Izmirlian G, Kramer BS. The National Lung Screening Trial: results stratified by demographics, smoking history, and lung cancer histology. Cancer. 2013;119(22):3976-83.

3. Menzies D. The case for a worldwide ban on smoking in public places. Curr Opin Pulm Med. 2011;17(2):116-22.

4. Coleman T, Agboola S, Leonardi-Bee J, Taylor M, McEwen A, McNeill A. Relapse prevention in UK stop smoking services: current practice, systematic reviews of effectiveness and cost-effectiveness analysis. Health Technol Assess. 2010;14(49):1-152. iii-iv
5. National Centre for Smoking Cessation and Training [http://www.ncsct.co. uk/index.php]. Accessed 18 Sept 2016.

6. Stop smoking services [https://www.nice.org.uk/guidance/ph10]. Accessed 18 Sept 2016.

7. Piné-Abata H, McNeill A, Murray R, Bitton A, Rigotti N, Raw M. A survey of tobacco dependence treatment services in 121 countries. Addiction. 2013;108(8):1476-84

8. Molyneux A. Nicotine replacement therapy. BMJ. 2004;328(7437):454-6.

9. Settle EC. Bupropion sustained release: side effect profile. J Clin Psychiatry. 1998:59(suppl 4):32-6.

10. McNeil JJ, Piccenna L, loannides-Demos LL. Smoking cessation-recent advances. Cardiovasc Drugs Ther. 2010;24(4):359-67.

11. Mills EJ, Wu P, Lockhart I, Thorlund K, Puhan M, Ebbert JO. Comparisons of high-dose and combination nicotine replacement therapy, varenicline, and bupropion for smoking cessation: a systematic review and multiple treatment meta-analysis. Ann Med. 2012;44(6):588-97.

12. Leaviss J, Sullivan W, Ren S, Everson-Hock E, Stevenson M, Stevens JW, Strong M, Cantrell A. What is the clinical effectiveness and costeffectiveness of cytisine compared with varenicline for smoking cessation? A systematic review and economic evaluation. Health Technol Assess. 2014;18(33):1-120.

13. Cahill K, Stevens S, Perera R, Lancaster T. Pharmacological interventions for smoking cessation: an overview and network meta-analysis. Cochrane Database Syst Rev. 2013;5:CD009329.

14. Owens DK, Qaseem A, Chou R, Shekelle P. High-value, cost-conscious health care: concepts for clinicians to evaluate the benefits, harms, and costs of medical interventions. Ann Intern Med. 2011;154(3):174-80.

15. Athanasakis K, Igoumenidis M, Karampli E, Vitsou E, Sykara G, Kyriopoulos J. Cost-effectiveness of varenicline versus bupropion, nicotine-replacement therapy, and unaided cessation in Greece. Clin Ther. 2012;34(8):1803-14.

16. Lutz MA, Lovato P, Cuesta G. Cost analysis of varenicline versus bupropion, nicotine replacement therapy, and unaided cessation in Nicaragua. Hosp Pract (Minneap). 2012:40(1):35-43.

17. Mahmoudi M, Coleman C, Sobieraj D. Systematic review of the costeffectiveness of varenicline vs. bupropion for smoking cessation. Int J Clin Pract. 2012;66(2):171-82.

18. Cornuz J, Gilbert A, Pinget C, McDonald P, Slama K, Salto E, Paccaud F. Cost-effectiveness of pharmacotherapies for nicotine dependence in primary care settings: a multinational comparison. Tob Control. 2006; 15(3):152-9.

19. Luce BR, Kramer JM, Goodman SN, Connor JT, Tunis S, Whicher D, Schwartz JS. Rethinking randomized clinical trials for comparative effectiveness research: the need for transformational change. Ann Intern Med. 2009;151(3):206-9.

20. Revicki DA, Frank L. Pharmacoeconomic evaluation in the real world. PharmacoEconomics. 1999:15(5):423-34.

21. van Staa T-P, Leufkens $H G$, Zhang $B$, Smeeth $L$. A comparison of cost effectiveness using data from randomized trials or actual clinical practice: selective cox-2 inhibitors as an example. PLoS Med. 2009;6(12):e1000194.

22. Coyle K, Coyle D, Lester-George A, West R, Nemeth B, Hiligsmann M, Trapero-Bertran M, Leidl R, Pokhrel S. Development and application of an economic model (EQUIPTMOD) to assess the impact of smoking cessation. Addiction. 2017;

23. World Medical Association Declaration of Helsinki. Ethical principles for medical research involving human subjects. Bull World Health Organ. 2001;79(4):373-4.

24. Heatherton TF, Kozlowski LT, Frecker RC, Fagerstrom KO. The Fagerstrom test for nicotine dependence: a revision of the Fagerstrom tolerance questionnaire. Br J Addict. 1991;86(9):1119-27.

25. UK Government. English indices of deprivation 2015. https://www.gov.uk/ government/statistics/english-indices-of-deprivation-2015. Accessed 29 Dec 2016.

26. Payne R, Harding S, Murray D, Soutar D, Baird D, Glaser A, Channing I, Welham S, Gilmour A, Thompson R. GenStat release 11 reference manual, part 2 directives. Hemel Hempstead, UK: VSN International; 2008.

27. Pokhrel S, Evers S, Leidl R, Trapero-Bertran M, Kalo Z, De Vries H, Crossfield A, Andrews F, Rutter A, Coyle K. EQUIPT: protocol of a comparative effectiveness research study evaluating cross-context transferability of economic evidence on tobacco control. BMJ Open. 2014;4(11):e006945 
28. Walker N, Van Woerden HC, Kiparoglou V, Yang Y, Robinson H, Croghan E. Gender difference and effect of pharmacotherapy: findings from a smoking cessation service. BMC Public Health. 2016;16(1):1038.

29. Graham AL, Chang Y, Fang Y, Cobb NK, Tinkelman DS, Niaura RS, Abrams DB, Mandelblatt JS: Cost-effectiveness of internet and telephone treatment for smoking cessation: an economic evaluation of The iQUITT Study. Tobacco control 2012:tobaccocontrol-2012-050465.

30. Bauld L, Bell K, McCullough L, Richardson L, Greaves L. The effectiveness of NHS smoking cessation services: a systematic review. J Public Health (Oxf). 2010;32(1):71-82.

31. Pirie PL, McBride CM, Hellerstedt W, Jeffery RW, Hatsukami D, Allen S, Lando H. Smoking cessation in women concerned about weight. Am J Public Health. 1992;82(9):1238-43.

32. Bolin K, Wilson K, Benhaddi H, De Nigris E, Marbaix S, Mork A-C, Aubin H-J. Cost-effectiveness of varenicline compared with nicotine patches for smoking cessation-results from four European countries. The European Journal of Public Health. 2009;19(6):650-4.

Submit your next manuscript to BioMed Central and we will help you at every step:

- We accept pre-submission inquiries

- Our selector tool helps you to find the most relevant journal

- We provide round the clock customer support

- Convenient online submission

- Thorough peer review

- Inclusion in PubMed and all major indexing services

- Maximum visibility for your research

Submit your manuscript at www.biomedcentral.com/submit
C) Biomed Central 